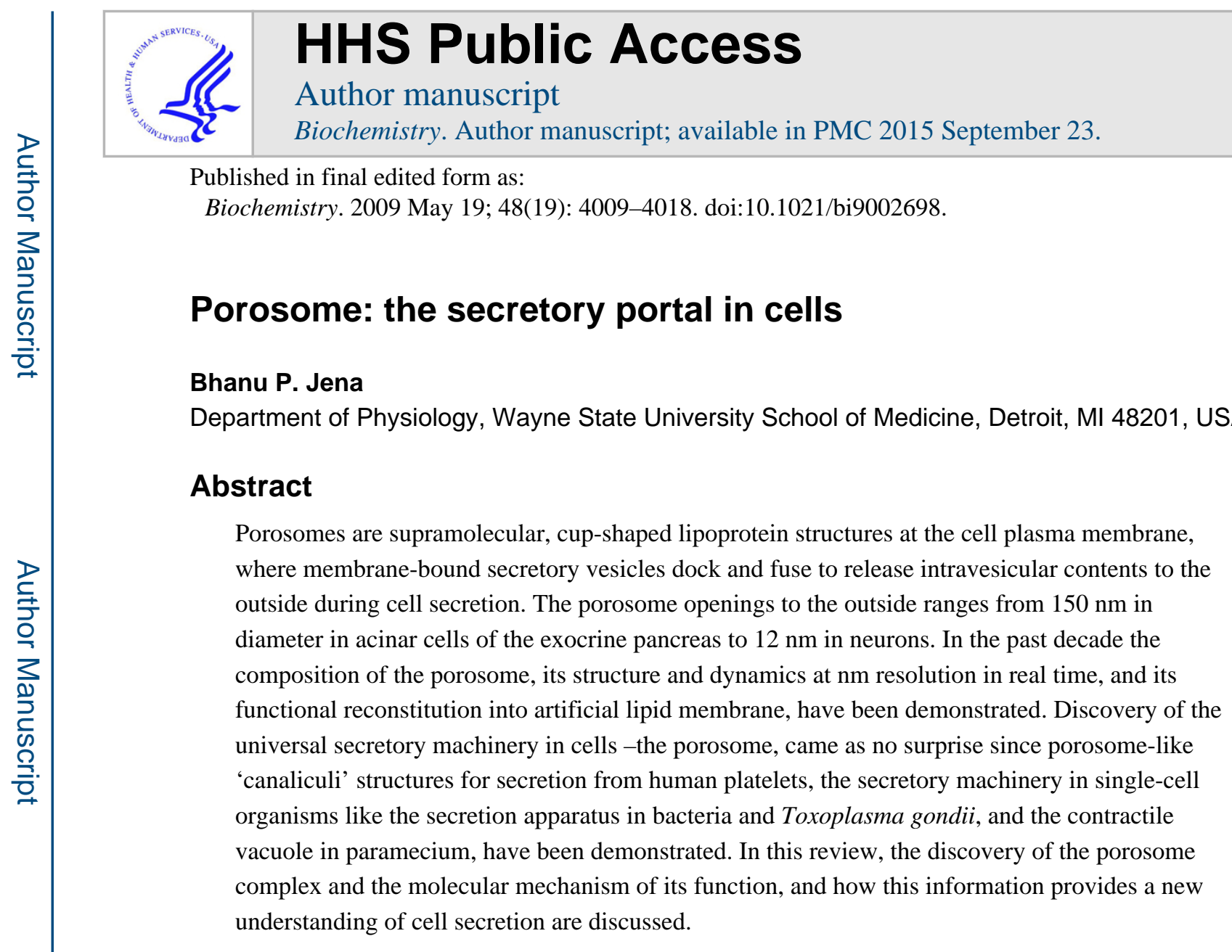

All life processes are governed at the chemical level and therefore knowledge of how single
molecules are arranged and interact provides a fundamental understanding of Nature. An
aspect of molecular interactions is the assembly of supramolecular structures for the purpose
of performing specific cellular functions. Secretion for example, is a fundamental cellular
process responsible for numerous physiological activities in living organisms, such as
neurotransmission and the release of hormones and digestive enzymes. Secretory defects in
cells are responsible for a host of debilitating diseases, and hence this field has been the
subject of intense study for over half a century. Only in the past decade using atomic force
microscopy (AFM), discovery of the 'porosome', a supramolecular structure at the cell
plasma membrane measuring only a few nanometers, and its determination as the universal
secretory machinery in cells, finally provides a molecular understanding of cell secretion. In
cells, secretory products packed and stored in membranous sacs or vesicles, dock and
establish continuity at the base of plasma membrane-associated structures called porosomes
(Fig. 1) (1-9), to release their contents to the outside. The subsequent isolation of the
porosome, and the determination of its biochemical composition, its structure and dynamics
at nm resolution and in real time, and its functional reconstitution into artificial lipid
membrane (1-9) has greatly advanced our understanding of the secretory process in cells,
echoed by a number of investigators (10-32). It is fascinating how even single-cell
organisms have developed such specialized secretory machinery, like the secretion apparatus

"Correspondence: Bhanu P. Jena, Ph.D., D. Sc., (hon. doct. mult.), George E. Palade University Professor, Distinguished Professor, Department of Physiology, Wayne State University School of Medicine, 5245 Scott Hall, Detroit, MI 48201, USA. Phone:

313-577-1532, Fax: 313-993-4177, bjena@med.wayne.edu, Web: http://www.med.wayne.edu/physiology/facultyprofile/jena. 
of Toxoplasma gondii (Fig. 1F) (33), the contractile vacuole in paramecium (34), and the secretory structures in bacteria (35). Hence it comes as no surprise that mammalian cells have evolved such highly specialized and sophisticated cup-shaped structures - the porosome complex, or the porosome-like 'canalicular systems' in human platelets (Fig. 1E) $(36,37)$, for the precise and regulated expulsion of intravesicular contents from cells, from neuro transmission, to the secretion of hormones from endocrine cells, and digestive enzymes from the exocrine pancreas. In every case, secretory vesicles have a much larger surface area, severalfold larger than that of the porosome complex. During cell secretion, the porosome opening dilates to enable release of sectory products, returning to its resting size following completion of secretion. According to conventional belief, if secretory vesicles were to completely merge at the porosome, it would result in a total obliteration of the porosome complex, since surface area of secretory vesicles are several-fold larger than that of the porosome. Studies discussed in this review demonstrate that secretory vesicles transiently dock and fuse at the base of porosomes to expel intravesicular contents during cell secretion (Fig. 2). This mechanism is in contrast to the general belief that in mammalian cells, secretory vesicles completely merge at the cell plasma membrane, resulting in passive diffusion of vesicular contents to the cell exterior. Furthermore, a major logistical problem with complete merging of secretory vesicle membrane at the cell plasma membrane is the presence of partially empty vesicles following cell secretion.

The establishment of continuity between the secretory vesicle membrane and the porosome membrane requires the participation of specific membrane proteins called SNAREs (38-42). At the nerve terminal for example, target membrane proteins SNAP-25 (38) and syntaxin (39), collectively called t-SNAREs present at the base of the neuronal porosome complex, and synaptic vesicle-associated membrane protein v-SNARE (40), are involved in fusion of synaptic vesicles at the porosome base $(41,42)$. In order to understand SNARE-induced membrane fusion, a comprehension of the interaction and assembly of membrane-associated $\mathrm{v}$-SNARE and t-SNARE proteins, is required. Solubility problems have precluded the generation of 3D crystals of membrane-associated t-/v-SNARE complexes. However, when the membrane-associated hydrophobic domain of the t-SNARE protein syntaxin and the vSNARE protein VAMP (vesicle-associated membrane protein) were truncated, the proteins were rendered soluble, allowing 3D crystallization of the truncated t-/v-SNARE complex and the determination of its structure at $2.4 \AA$ (43). Unfortunately, it soon became apparent that in the absence of membrane, t-SNAREs and v-SNARE interact differently (44), thereby precluding formation of the physiologically relevant t-/v-SNARE complex in cells. The structure and arrangement of membrane-associated t-/v-SNARE complex were first determined using AFM (44). Results from the study demonstrate that t-SNAREs and vSNARE, when present in opposing bilayers, interact in a circular array to form ring complexes or channels, each measuring a few nanometers (Fig. 3) (44). The size of the ring complex is directly proportional to the curvature of the opposing bilayers, and in the presence of calcium, the SNARE ring-complex enables the establishment of continuity between the opposing bilayers. In contrast, in the absence of membrane association, soluble $\mathrm{v}$ - and t-SNAREs fail to assemble in such organized rings, nor establish continuity between the opposing bilayers $(44,45)$. Once v-SNARE and t-SNAREs residing in opposing bilayers meet, the resulting SNARE complex overcomes the repulsive forces between opposing 
bilayers, bringing them within a distance of 2.8-3 $\AA$. This allows calcium bridging of the opposing phospholipids head-groups and leads to local dehydration and membrane fusion (46-48).

In this review, the discovery of the porosome as the universal secretory machinery or portal in cells, its structure, dynamics, its isolation, composition, its functional reconstitution in artificial lipid membrane, and the molecular mechanism of SNARE-induced fusion of secretory vesicle at the porosome base, is discussed. The article is primarily focused on porosome in both slow and fast secretory cells namely, the acinar cell of the exocrine pancreas and neurons, respectively. Additionally, studies on neuronal SNAREs, provide an understanding of the molecular mechanism of SNARE-induced membrane fusion, i.e., the fusion of synaptic vesicles at the porosome base in neurons. The discovery of the porosome complex, and the new insights into its structure-function, has resulted in a paradigm shift in our understanding of cell secretion, is further discussed in this review.

\section{Discovery of the 'porosome' - the universal secretory machinery in cells}

Porosomes were first discovered in acinar cells of the exocrine pancreas (1). Exocrine pancreatic acinar cells are polarized secretory cells possessing an apical and a basolateral end. This well characterized cell synthesizes digestive enzymes, which are stored within $0.2-1.2 \mu \mathrm{m}$ in diameter membranous sacs or secretory vesicles called zymogen granules (ZG), located apically. Following a secretory stimulus, ZG's dock and fuse with the apical plasma membrane to release their contents to the outside. In contrast to neurons, where secretion of neurotransmitters occurs in milliseconds, the pancreatic acinar cells secrete digestive enzymes over minutes following a secretory stimulus. Being a slow secretory cell, pancreatic acinar cells were considered ideal for investigating the molecular steps involved in cell secretion. In the mid 1990's, AFM studies on live pancreatic acinar cells were performed to evaluate at high resolution, the structure and dynamics of the apical plasma membrane surface, in both resting and stimulated cells. To our surprise, isolated live pancreatic acinar cells in physiological buffer, when imaged using the AFM (1), reveal new cellular structures at the apical plasma membrane, in the form of 'pits' and 'depressions'. Circular 'pits' measuring 0.4-1.2 $\mu \mathrm{m}$, and typically 3-4 'depressions' or pores (Fig. 1A,B) measuring 100-180 nm in diameter within each pit, are found at the apical end of the cell. The basolateral membrane in acinar cells is devoid of such pit or depression structures. High-resolution AFM images of depressions in live acinar cells further reveal a cone-shaped morphology, and with the depth of each cone measures $15-35 \mathrm{~nm}$. Similarly, examination of resting growth hormone (GH) secretory cells of the pituitary gland (3), neurons, astrocytes, $\beta$-cells of the endocrine pancreas, mast cells, and chromaffin cells of the adrenal medulla $(4,7,15,16)$, each show evidence of the presence of depressions at the cell plasma membrane.

Exposure of pancreatic acinar cells to a secretagogue (mastoparan/Mast.7) results in a timedependent increase (25-45\%) in both the diameter and relative depth of depressions (Fig. 2). Studies demonstrate that depressions return to their resting size on completion of cell secretion (Fig. 2), with no demonstrable change in pit size $(1,2)$. Enlargement of depression diameter and relative depth following exposure to the secretagogue, correlates with secretion. Aditionally, exposure of pancreatic acinar cells to cytochalasin B, a fungal toxin 
that inhibits actin polymerization and cell secretion, results in a $15-20 \%$ decrease in depression size and a consequent 50-60\% loss in secretion (1). Results from these studies suggested depressions to be the fusion pores in pancreatic acinar cells. These studies further demonstrated the involvement of actin in regulation of both the structure and function of depressions. Similarly, depression in resting GH cells measuring $154 \pm 4.5 \mathrm{~nm}$ (mean $\pm \mathrm{SE}$ ) in diameter, results in a $40 \%$ increase in depression diameter $(215 \pm 4.6 \mathrm{~nm}$; $\mathrm{p}<0.01)$, with no appreciable change in pit size, following stimulation of secretion (3). The enlargement of depression diameter during cell secretion and decrease of depression diameter and loss of secretion following exposure to actin depolymerizing agents (3), suggested these to be secretory pores or portholes. However, the direct determination that depressions are indeed the portholes via which secretory products are released from cells, finally came from immuno-AFM studies. Localization at depressions of gold-conjugated antibody to secretory proteins, provided direct evidence that secretion occurs through depressions $(2,3)$. Another example came from zymogen granules contain the starch digesting enzyme amylase. AFM micrographs of the localization of gold-tagged amylase-specific antibodies at depressions, following stimulation of cell secretion $(2,5)$, demonstrated depressions to be the cells secretory portholes. Similarly, in somatotrophs of the pituitary gland, gold-tagged growth hormone-specific antibody is found to selectively localize at depressions following stimulation of secretion (3), and established them to be the secretory pores or the secretory portal in these cells. Over the years, the term "fusion pore" has been loosely referred to plasma membrane dimples that originate following a secretory stimulus, or to the continuity or channel established between opposing lipid bilayer during membrane fusion. Hence for clarity, the term "porosome" was assigned to depressions, which are permanent structures at the cell plasma membrane where secretory vesicles dock and fuse to expel intra-vesicular contents to the outside.

The morphology of the porosome complex facing the cytosolic compartment of the cell in exocrine pancreas (5), and in neurons (4), have also been determined at near nm resolution in sub-cellular fractions. AFM studies on isolated plasma membrane preparations in near physiological solution, reveal scattered circular disks measuring $0.5-1 \mu \mathrm{m}$ in diameter, with inverted cup-shaped structures (5). The inverted cups at the cytosolic compartment of isolated pancreatic plasma membrane preparations range in height from 10-15 nm. On several occasions, ZG's ranging in size from $0.4-1 \mu \mathrm{m}$ in diameter, were observed in association with one or more of these inverted cups, suggesting them to be porosomes. To further confirm that the cup-shaped structures are porosomes, immuno-AFM studies were performed. Target membrane proteins, SNAP-23/-25 (38) and syntaxin (t-SNARE) (39) and secretory vesicle-associated membrane protein v-SNARE or VAMP (40), are part of the conserved protein complex involved in the fusion of opposing bilayers in the presence of calcium $(41,42,44,61-63)$. If porosomes are the secretory sites for vesicle docking and fusion, then plasma membrane-associated t-SNAREs should localize at the base of the structure facing the cytosol. The t-SNARE protein SNAP-23 had previously been reported in pancreatic acinar cells (64). A polyclonal monospecific SNAP-23 antibody recognizing a single $23 \mathrm{kDa}$ protein in immunoblots of resolved pancreatic plasma membrane fraction, demonstrated selective localization to the porosome base. These results confirmed the inverted cups in inside-out isolated pancreatic plasma membrane preparations, to be 
porosomes, where secretory vesicles dock and fuse to release their contents during cell secretion (5).

The morphology of the pancreatic porosome complex has been further evaluated using transmission electron microscopy (TEM) (6). TEM studies confirm the porosome to possess a cup-shaped structure, with similar dimensions as determined from AFM measurement. Additionally, TEM micrographs demonstrate pancreatic porosomes to display a basket-like morphology, with three lateral and a number of vertically arranged ridges. A ring at the base of the complex is further identified (6), and is hypothesized to represent t-SNAREs or t-/vSNARE complexes organized in a circular array. Studies using full length recombinant SNARE proteins and artificial lipid membrane demonstrate that $\mathrm{t}$ and $\mathrm{v}$-SNAREs located in opposing bilayers interact in a circular array to form conducting channels in the presence of calcium (44). Earlier determination of the presence of SNAP-23 at the porosome base (5), suggests the circular arrangement of proteins could be t-SNAREs or t-/v-SNARE complex. In the past decade, a number of studies demonstrate the involvement of cytoskeletal proteins in cell secretion, some implicating their direct interaction with SNAREs (65-70).

Furthermore, actin and microtubule-based cytoskeleton has been implicated in intracellular vesicle traffic. Fodrin, which was previously implicated in exocytosis, has also been shown to directly interact with SNAREs (68). Studies demonstrate a-fodrin to regulate exocytosis via its interaction with the t-SNARE syntaxin family of proteins. The C-terminal region of syntaxin is known to interact with a-fodrin, a major component of the submembranous cytoskeleton. Similarly, vimentin filaments interact with SNAP23/25 and hence are able to control the availability of free SNAP23/25 for assembly of the t-/v-SNARE complex (66). All these findings suggest that vimentin, a-fodrin, actin, and SNAREs may be part of the porosome complex. Additional proteins such as v-SNARE, synaptophysin and myosin, may associate when the porosome establishes continuity with the secretory vesicle membrane. The globular tail domain of myosin V contains a binding site for VAMP, which is bound in a calcium independent manner (69). Further interaction of myosin $\mathrm{V}$ with syntaxin had previously been shown to require both calcium and calmodulin. It had also been suggested that VAMP, may act as a myosin $\mathrm{V}$ receptor on secretory vesicles, and regulate formation of the SNARE complex (68). Interaction of VAMP with synaptophysin and myosin V had also been reported (69). In agreement with these earlier findings, our studies $(4,5)$ demonstrate the association of SNAP-23, syntaxin 2, cytoskeletal proteins actin, a-fodrin, and vimentin, and calcium channels $\beta 3$ and a1c, together with the SNARE regulatory protein NSF, at the porosome complex (4-6). Additionally, chloride ion channels $\mathrm{ClC} 2$ and $\mathrm{ClC} 3$ were also identified as part of the porosome complex (4-6). Isoforms of a number of proteins identified as components of the porosome complex, have also been reported using 2D-BAC gels electrophoresis (6). Three isoforms each of the calcium ion channel and vimentin has been identified in porosomes (6). Using yeast two-hybrid analysis, studies further confirmed the presence and interaction of some of these proteins with t-SNAREs within the porosome complex (70).

The size and shape of the immunoisolated porosome complex have also been determined using both negative staining EM and AFM (6). The morphology of immunoisolated porosomes obtained using EM and AFM is almost identical, and super imposable (6). The immunoisolated porosome complex has also been structurally and functionally reconstituted 
into liposomes and bilayer membranes $(4,6)$. Transmission electron micrographs of pancreatic porosomes reconstituted into liposomes exhibit a 150-200 nm cup-shaped basketlike morphology, similar to what is observed in its native state when co-isolated with ZGs. To test the functionality of isolated porosomes obtained from exocrine pancreas or neurons, they were reconstituted into the lipid membrane of the electrophysiological setup (EPC9), and exposed to isolated ZGs or synaptic vesicles. Electrical activity of the reconstituted membrane as well as the transport of vesicular contents from the cis to the trans compartments of the bilayer chambers was then monitored. Results from these experiments demonstrate that the lipid membrane-reconstituted porosomes, are indeed functional $(4,6)$, demonstrating isolated secretory vesicle docking, fusion and the transfer of intravesicular contents from the cis to the trans compartment of the bilayer chamber. ZGs fuse with the porosome-reconstituted bilayer as demonstrated by an increase in capacitance and conductance, and a time-dependent transport of the ZG enzyme amylase from cis to the trans compartment of the bilayer chamber $(6,8)$. Amylase is detected using immunoblot analysis of the buffer in the $c$ is and trans chambers of the bilayer apparatus $(6,8)$. Chloride channel activity is also present in the reconstituted porosome complex, and the channel inhibitor 4,4'-diisothiocyanato-stilbene-2-2'-disulfonic acid (DIDS) inhibited current activity through the porosome-reconstituted bilayer, demonstrating its requirement for porosome function. Similarly, the structure and biochemical composition of the neuronal porosome complex (Fig. 1C,D), and the docking and fusion of synaptic vesicles at the structure has also been determined $(4,7)$.

In recent years, a number of laboratories $(11,49-51)$ have also demonstrated the presence of porosomes and their involvement in cell secretion from neurons, neuroendcrine cells, to acinar cells of the exocrine pancreas. Although at much lower resolution than AFM observations $(4,7,52)$, recent EM studies (49) of the presynaptic terminal, also demonstrate synaptic vesicles docked at $12-15 \mathrm{~nm}$ electron dense structures. Similarly in separate studies from three different laboratories using EM, AFM, and high molecular weight dyes, on pancreatic acinar cells $(11,50)$, and on gonadotrophs of the anterior pituitary gland (51), porosomes or fusion pores have been identified and implicated in cell secretion. In summary, these studies collectively demonstrate porosomes to be permanent supramolecular lipoprotein structures at the cell plasma membrane, where membrane-bound secretory vesicles transiently dock and fuse to release intravesicular contents to the outside $(8,9,16)$. Porosomes in exocrine pancreas and neuroendocrine cells measure 100-180 nm, and only a $20-35 \%$ increase in porosome diameter is demonstrated following the docking and fusion of $0.2-1.2 \mu \mathrm{m}$ in diameter secretory vesicles, suggesting that secretory vesicles "transiently" dock and fuse at the base of the porosome complex to release their contents to the outside. In mammalian cells, it has long been held that secretory vesicles completely merge at the cell plasma membrane allowing intra-vesicular contents to passively diffuse out of the cell during secretion. If secretory vesicles completely merge at the cell plasma membrane, the porosome structure would be totally obliterated, and the generation of partially empty vesicles following cell secretion would be inexplicable. With the discovery of the porosome and the transient docking, fusion, and dissociation of secretory vesicles at its base, the generation of partially empty vesicles following cell secretion can now be explained. In agreement, several studies from a number of laboratories report that "secretory granules are 
recaptured largely intact after stimulated exocytosis in cultured endocrine cells" (53), that "single synaptic vesicles fuse transiently and successively without loss of identity" (54), that "Zymogen granule exocytosis is characterized by long fusion pore openings and preservation of vesicle lipid identity" (55), and that the "number of secretory vesicles in growth hormone cells of the pituitary remain unchanged after secretion" (56). It is fascinating how even single cell organisms have developed specialized secretory structures, such as the secretion apparatus of Toxoplasma gondii, (Fig. 1F) (33), specialized secretory systems in bacterial cells (35), the trichocyst structure and its discharge and the contractile vacuole in paramecium (34). Therefore it is not surprising that mammalian cells have evolved such highly specialized and sophisticated structures, the porosomes, for the precise and regulated expulsion of intra-vesicular contents during cell secretion, from neuro transmission (Fig. 1C,D), endocrine secretion, to the secretion of digestive enzymes from the exocrine pancreas (Fig. 1A,B), or the specialized porosome-like "canaliculi system" for secretion from human platelets (Fig. 1E) $(36,37)$.

\section{Participation of SNAREs in secretory vesicle fusion at the porosome base}

V-SNARE and t-SNAREs need to reside in opposing membrane to appropriately interact and establish continuity across opposing bilayers (44). Purified recombinant $t$ and v-SNARE proteins, when applied to a lipid membrane, form globular complexes and do not alter membrane current. In contrast, when t-SNAREs and v-SNARE in opposing bilayers are exposed to each other in calcium buffer, they interact and organize in a circular array, forming channel-like structures (Fig. 3). These channels are conducting, since some vesicles having discharged their contents appear flat, measuring only $10-15 \mathrm{~nm}$ in height, compared to their $40-60 \mathrm{~nm}$ when filled. Since the t-/v-SNARE complex is established between opposing bilayers, their organization is visible only following vesicle discharge, or when vesicles are dislodged from the complex by the AFM cantilever tip (Fig. 3F). Further determination of the establishment of continuity between opposing membrane via the t-/vSNARE ring complex, is demonstrated from electrophysiological assays (Fig. 3A,B). In the EPC9 electrophysiological setup (Fig. 3A), the conductance and capacitance of a v-SNAREreconstituted bilayer membrane is monitored following exposure to a t-SNARE reconstituted vesicles (Fig. 3B). Nystatin, in the presence of ergosterol, forms a cationconducting channel in lipid membranes (57-60). In calcium buffer, lipid vesicles reconstituted with t-SNAREs, nystatin, and ergosterol, when added to the cis compartment of the bilayer chamber, fuse with the v-SNARE reconstituted membrane. When vesicles containing nystatin and ergosterol incorporate into an ergosterol-free bilayer membrane of the EPC9 setup, a current spike is observed since the nystatin channel collapses as ergosterol diffuses into the lipid membrane (57-60). As a positive control, a $\mathrm{KCl}$ gradient tests the ability of vesicles to fuse at the lipid membrane $(410 \mathrm{mM}$ cis: $150 \mathrm{mM}$ trans). The $\mathrm{KCl}$ gradient provides a driving force for vesicle incorporation that is independent of the influence of SNARE proteins. When t-SNARE vesicles are exposed to v-SNARE reconstituted bilayers, vesicles fuse, which is observed as a current spike, and an increase in membrane capacitance (44). These studies demonstrate that in absence of membrane, even full-length t-SNAREs and v-SNARE interact differently (44), and as a result are incapable of organizing into the physiologically relevant t-/v-SNARE ring conformation. These results 
called for a reevaluation of the $2.4 \AA$ atomic coordinates determined using x-ray crystallography on crystals of the t-/v-SNARE complex obtained from truncated, and nonmembrane associated $t$ and $v$-SNARE proteins (43). Therefore determination of the atomic structure of membrane-associated full-length SNAREs and their complexes remain a major challenge. At the moment, cryo EM and electron crystallography are the most logical approaches, and is being carried out in the author's laboratory.

So what is the molecular mechanism of SNARE ring complex formation, and what dictates its size? Due to the rapid formation of the t-/v-SNARE ring complex between opposing bilayers, direct observation of its formation at ultra high resolution has been practically impossible. Hypothetically however, the process may involve a progressive recruitment of $\mathrm{t}-/ \mathrm{v}-\mathrm{SNARE}$ pairs as the opposing vesicles are pulled toward each other, until a complete ring is established, preventing further recruitment of t-/v-SNARE pairs (Fig. 3J). The size of the membrane-associated $\mathrm{t}-\mathrm{v}$-SNARE complex however, has been found to reflect the vesicle curvature. The larger the vesicle, the smaller is its curvature, and the larger is the t-/v-SNARE complex (Fig. 3E-G). Studies using t-SNARE-reconstituted membrane when exposed to v-SNARE-reconstituted liposomes of different sizes, demonstrate that the size of the t-/v-SNARE ring complex is directly proportional to the proteoliposome size (61).

\section{Disassembly of the SNARE complex}

Studies demonstrate that the soluble $N$-ethylmaleimide-sensitive factor (NSF) an ATPase, disassembles the t-/v-SNARE complex in presence of ATP (62). This study was also the first confirmation by direct physical observation that NSF-ATP alone can lead to SNARE complex disassembly. Using purified recombinant NSF, and $t$ and v-SNARE-reconstituted liposomes, the disassembly of the t-/v-SNARE complex was examined. Lipid vesicles ranging in size from $0.2-2 \mu \mathrm{m}$ were reconstituted with either t-SNAREs or v-SNARE. Kinetics of association and dissociation of t-SNARE and v-SNARE-reconstituted liposomes in solution, in the presence or absence of NSF, ATP, and AMP-PNP (the non-hydrolyzable ATP analogue), was monitored by right angle light scattering. Addition of NSF and ATP to the $\mathrm{t} / \mathrm{v}$-SNARE-vesicle mixture led to a rapid and significant increase in intensity of light scattering, suggesting rapid disassembly of the SNARE complex and the consequent dissociation of vesicles (62). Dissociation of t-/v-SNARE vesicles occurred on a logarithmic scale that was expressed by a first order reaction kinetics, with a rate constant $\mathrm{k}=1.1 \mathrm{~s}^{-1}$. To further determine whether NSF-induced dissociation of $t$ and v-SNARE vesicles is energy driven, experiments were performed in the presence and absence of ATP and AMP-PNP. No significant change with NSF alone, or in presence of NSF-AMP-PNP, was observed demonstrating that $\mathrm{t}-/ \mathrm{v}$-SNARE disassembly is an enzymatic and energy-driven process.

The role of NSF-ATP in the disassembly of the t-/v-SNARE complex has also been confirmed using an immunochemical approach. It has been demonstrated that v-SNARE and t-SNAREs formed a very stable and an SDS-resistant complex (71). NSF binds to SNAREs and forms a stable complex when locked in the ATP-bound state (ATP-NSF). Thus, in the presence of ATP+EDTA, VAMP antibody has been demonstrated to be able to coprecipitate the NSF-SNARE complex (71). Therefore, when $t$ and v-SNARE vesicles were mixed in the presence or absence of ATP, NSF, NSF+ATP, or NSF+AMP-PNP, followed 
by SDS-PAGE, electro-transferred to nitrocellulose membrane and immunoblot analysis using syntaxin-1 specific antibody, t-/v-SNARE disassembly was found to be complete only in the presence of NSF-ATP. Direct observation of the t-/v-SNARE complex disassembly was further assessed using AFM (62). When purified recombinant t-SNAREs and v-SNARE in opposing bilayers interact and self-assemble to form supramolecular ring complexes, they disassembly when exposed to recombinant NSF and ATP, as observed at nm resolution using AFM (62). Furthermore, close examination at near nanometer resolution, NSF-ATPinduced disassembly of the SNARE complex by AFM, demonstrated NSF to function as a right-handed molecular motor (64).

\section{Circular dichroism spectroscopy on SNARE assembly-disassembly}

Circular dichroism (CD) spectroscopy confirms the overall structure of membraneassociated SNAREs and SNARE complexs to be different from those formed in the absence of membrane (45). This study further confirms NSF-ATP to be sufficient for SNARE disassembly (Fig. 3H,I). The overall secondary structural content of full-length neuronal vSNARE and t-SNAREs, and the t-/v-SNARE complex, both in suspension and membraneassociated, were determined by CD spectroscopy using an Olis DSM 17 spectrometer (45). CD spectroscopy reveals that $\mathrm{v}$-SNARE in buffered suspension, when incorporated into liposomes, exhibit reduced folding (Table 1). This loss of secondary structure following incorporation of full-length v-SNARE in membrane may result from self-association of the hydrophobic regions of the protein in the absence of membrane. When incorporated into liposomes, v-SNARE may freely unfold without the artifactual induction of secondary structure, as reflective of the lack in CD signals at 208 and $222 \mathrm{~nm}$, distinct for a-helical content. The t-SNAREs show clearly defined peaks at both these wavelengths, consistent with a higher degree of helical secondary structures formed both in buffered suspension and in membrane, at ca. 66 and 20\%, respectively (Table 1). Again, similar to v-SNARE the membrane-associated t-SNAREs exhibit less helical content than in suspension. Similarly, there appears to be a dramatic difference in the CD signal observed in t-/v-SNARE complexes in suspension, and those complexes that are formed when membrane-associated SNAREs interact. Interestingly, there is no increase of secondary structure upon complex formation. Rather, the $\mathrm{CD}$ spectra of the complexes are identical to a combination of individual spectra. Moreover, membrane associated t-/v-SNAREs are less folded than the

purified SNARE complex. These data support previous AFM results that lipid is required for proper arrangement of the SNARE proteins in membrane fusion. Addition of NSF to the $\mathrm{t}-/ \mathrm{v}-\mathrm{SNARE}$ complex results in an increase in the unordered fraction (Table 1), which may be attributed to an overall disordered secondary structure of the NSF, and not necessarily unfolding of the t-/v-SNARE complex. In contrast, activation of NSF by the addition of ATP almost completely abolishes all a-helical content within the multi-protein complex. This direct observation of the helical unfolding of the SNARE complex using CD spectroscopy under physiologically relevant conditions (i.e. in membrane-associated SNAREs), confirms AFM reports on NSF-ATP-induced t-/v-SNARE complex disassembly (62). In further agreement with previously reported studies using the AFM, the consequence of ATP addition to the t-/v-SNARE-NSF complex is disassembly, regardless of whether the $\mathrm{t}-\mathrm{v}$-SNARE+NSF complex is membrane-associated or in buffered suspension. In earlier AFM studies, $0.16-0.2 \mu \mathrm{g} / \mathrm{ml}$ of SNARE proteins were used, as opposed to the 800-1000 
$\mu \mathrm{g} / \mathrm{ml}$ protein concentration required for the current CD studies. To determine if t-SNARE and $\mathrm{v}$ SNARE interact differently at higher protein concentrations, both membraneassociated and in-suspension $\mathrm{v}$ and t-SNARE complexes used in CD studies, were imaged using the AFM. In confirmation, results from the CD study demonstrated the formation of $\mathrm{t}-\mathrm{v}$-SNARE ring complexes, only when t-SNARE-liposomes are exposed to v-SNAREliposomes. Hence, higher SNARE protein concentrations are without influence on the membrane-directed self-assembly of the SNARE complex (45). In summary, the CD results demonstrate that v-SNARE in suspension or when incorporated into liposomes, exhibit reduced folding. Similarly, t-SNAREs which exhibit clearly defined peaks at CD signals of 208 and $222 \mathrm{~nm}$ wavelengths, consistent with a higher degree of helical secondary structure in both the soluble and liposome-associated forms, exhibit reduced folding when membraneassociated. ATP-induced activation of NSF bound to the t-/v-SNARE complex, results in disassembly of the SNARE complex, eliminating all a-helices within the structure. These studies are a further confirmation of earlier reports (62) that NSF-ATP is sufficient for the disassembly of the t-/v-SNARE complex.

\section{SNAREs bring opposing bilayers closer, enabling calcium bridging and membrane fusion}

$\mathrm{X}$-ray diffraction patterns of non-reconstituted vesicles and $\mathrm{t}$ and v-SNARE-reconstituted vesicles in the absence and presence of $5 \mathrm{mM} \mathrm{Ca}^{2+}$ have been performed in the $2-4 \AA$ diffraction range (46), having broad pattern spanning 2 ranges approximately $23-48^{\circ}$ or $\mathrm{d}$ values of 3.9-1.9 $\AA$ with sharp drop off intensity on either sides of the range. A broad feature of the diffractogram indicates a multitude of contacts between atoms of one vesicle as well as between different vesicles. However, two broad peaks are visible on the diffractogram, the stronger being at $3.1 \AA$ and a weaker one at $1.9 \AA$, indicating that the greatest number of contacts between them have these two separating distances. The addition of $\mathrm{Ca}^{2+}$ or the incorporation of SNAREs at the vesicles membrane, or both, influence both peaks within the 2.1-3.3 $\AA$ intensity range. The influence of $\mathrm{Ca}^{2+}$, SNAREs, or both is more visible on a peak positioned at $3.1 \AA$ in the form of an increased Imax of arbitrary units and 2. This increase of Imax at the $3.1 \AA$ can be explained in terms of increased vesicle pairing and/or a decrease in distance between apposed vesicles. Incorporation of $t$ and v-SNARE proteins at the vesicle membrane allows for tight vesicle-vesicle interaction, demonstrated again as an Imax shifts to $30.5^{\circ}$ or $2.9 \AA$ from $3.1 \AA . \mathrm{Ca}^{2+}$ and SNAREs work in a manner that induces a much higher increase of peak intensity with appearance of shoulders at $2.8 \AA$.

Hydrated calcium $\left[\mathrm{Ca}\left(\mathrm{H}_{2} \mathrm{O}\right)_{n}\right]^{2+}$ has more than one shell around it, and the first hydration shell around the $\mathrm{Ca}^{2+}$ has six water molecules in an octahedral arrangement (37). Calcium drives SNARE-induced fusion of opposing bilayers $(46,47)$. SNARE interactions allow opposing bilayers to come close within a distance of approximately $2.8 \AA$. Using light scattering and $\mathrm{x}$-ray diffraction experiments involving SNARE-reconstituted liposomes, it has become clear that fusion proceeds only when $\mathrm{Ca}^{2+}$ ions are available between the $t$ and v-SNARE-apposed bilayers (47). Since t-SNAREs and v-SNARE in opposing bilayers interact in a circular array to form conducting channels in the presence of calcium (44), it would necessitate $\mathrm{Ca}^{2+}$ ions to be present between the SNARE-apposed bilayers, to allow bridging of the opposing membranes. Once calcium forms such a bridge, it can no longer hold its water shells, leading to water expulsion, membrane destabilization, and fusion. To 
confirm results of x-ray diffraction studies, and further test the above hypothesis, atomistic molecular dynamic simulations in the isobaric-isothermal ensemble using hydrated dimethylphosphate anions (DMP-) and calcium cations, were performed (48). DMP was chosen for the study since it represented the smallest molecular fragment of typical membrane phospholipids that retained properties of the phospholipid head-group, while providing a significant reduction in the computational complexity and enhance accuracy of the study. Furthermore, the strategy of using the DMP rather than full phospholipids in the simulation facilitates the search for spontaneously formed $\mathrm{Ca}^{2+}$-phospholipid structures, which may bridge the head groups of opposing phospholipids bilayers. Results from the simulation study clearly demonstrated that DMP and calcium form DMP-Ca ${ }^{2+}$ complexes and the consequent removal of water, supporting the hypothesis. As a result of $\mathrm{Ca}^{2+}$-DMP self-assembly, the distance between anionic oxygens between the two DMP molecules is reduced to $2.92 \AA$ (48), which is in agreement with the $2.8 \AA$ SNARE-induced apposition established between opposing lipid bilayers, reported from $\mathrm{x}$-ray diffraction measurements (46). The above findings provide for the first time, a molecular understanding of SNAREinduced membrane fusion in cells.

\section{CONCLUSION}

In this article, the current understanding of the molecular machinery and mechanism of secretion in cells is presented. Porosomes are specialized plasma membrane structures universally present in secretory cells, from exocrine and endocrine cells, to neuroendocrine cells and neurons. Since porosomes in exocrine and neuroendocrine cells measure 100-180 $\mathrm{nm}$, and only a $20-35 \%$ increase in porosome diameter is demonstrated following the docking and fusion of 0.2-1.2 $\mu \mathrm{m}$ in diameter secretory vesicles, it is concluded that secretory vesicles "transiently" dock and fuse at the base of the porosome complex to release their contents to the outside. This is in contrast to the general belief that in mammalian cells, secretory vesicles completely merge at the cell plasma membrane, resulting in passive diffusion of vesicular contents to the cell exterior, and the consequent retrieval of excess membrane by endocytosis at a later time. Additionally, a major logistical problem with the concept of complete merging of secretory vesicle membrane at the cell plasma membrane is an explanation of the generation of partially empty vesicles following cell secretion. It is fascinating how even single-cell organisms have developed such specialized secretory machinery, like the secretion apparatus of Toxoplasma gondii, the contractile vacuole in paramecium, and the secretory structures in bacteria. Hence it comes as no surprise that mammalian cells have evolved such highly specialized and sophisticated structures the porosome complex for cell secretion. The discovery of the porosome, and an understanding of its structure and dynamics at $\mathrm{nm}$ resolution and in real time in live cells, its composition, and its functional reconstitution in lipid membrane, has greatly advanced our understanding of cell secretion. It is evident that the secretory process in cells is a well coordinated, highly regulated, and a finely tuned biomolecular orchestra. Clearly, these findings could not have advanced without the AFM, and therefore this powerful tool has greatly contributed to a new understanding of the cell. The AFM has enabled the determination of live cellular structure-function at sub nanometer to angstrom resolution, in real time, contributing to the birth of the new field of NanoCellBiology. Future directions 
will involve an understanding of the protein distribution and their arrangement at atomic resolution, and a similar understanding of the structure of the t-/v-SNARE ring complex. Determination of the atomic structure of membrane-associated full-length SNAREs and their complexes, and of the neuronal porosome complex, will be accomplished using cryo EM and electron crystallography, and is currently being carried out in the author's laboratory.

\section{Acknowledgments}

FUNDING This work was supported by grants from, NSF CBET-0730768, NIH NS-39918, and Wayne State Univ. Research Enhancement Award (BPJ),

The author thanks the many students and collaborators who have participated in the various studies discussed in this article. Research in the authors laboratory was support by Grants from the NIH, NSF, and Wayne State University.

\section{ABBREVIATIONS}

SNARE $\quad N$-ethylmaleimide-sensitive factor (NSF)-attachment protein receptors

t-SNAREs target SNAREs

V-SNARE vesicle SNARE or VAMP or vesicle associated membrane protein

AFM atomic force microscopy

CD circular dichroism

ZG zymogen granule

GH growth hormone

\section{References}

1. Schneider SW, Sritharan KC, Geibel JP, Oberleithner H, Jena BP. Surface dynamics in living acinar cells imaged by atomic force microscopy: identification of plasma membrane structures involved in exocytosis. Proc Natl Acad Sci USA. 1997; 94:316-321. [PubMed: 8990206]

2. Cho SJ, Quinn AS, Stromer MH, Dash S, Cho J, Taatjes DJ, Jena BP. Structure and dynamics of the fusion pore in live cells. Cell Biol Int. 2002; 26:35-42. [PubMed: 11779219]

3. Cho S-J, Jeftinija K, Glavaski A, Jeftinija S, Jena BP, Anderson LL. Structure and dynamics of the fusion pores in live $\mathrm{GH}$-secreting cells revealed using atomic force microscopy. Endocrinology. 2002; 143:1144-1148. [PubMed: 11861542]

4. Cho WJ, Jeremic A, Rognlien KT, Zhvania MG, Lazrishvili I, Tamar B, Jena BP. Structure, isolation, composition and reconstitution of the neuronal fusion pore. Cell Biol Int. 2004; 28:699708. [PubMed: 15516328]

5. Jena BP, Cho SJ, Jeremic A, Stromer MH, Abu-Hamdah R. Structure and composition of the fusion pore. Biophys J. 2003; 84:1-7. [PubMed: 12524260]

6. Jeremic A, Kelly M, Cho SJ, Stromer MH, Jena BP. Reconstituted fusion pore. Biophys J. 2003; 85:2035-2043. [PubMed: 12944316]

7. Cho WJ, Jeremic A, Jin H, Ren G, Jena BP. Neuronal fusion pore assembly requires membrane cholesterol. Cell Biol Int. 2007; 31:1301-1308. [PubMed: 17703958]

8. Jena BP. Secretion machinery at the cell plasma membrane. Curr Opin Struct Biol. 2007; 17:437443. [PubMed: 17764925]

9. Jena BP. Molecular machinery and mechanism of cell secretion. Exp Biol Med. 2005; 230:307-319.

10. Jeremic A. Cell secretion: an update. J Cell Mol Med. 2008; 12:1151-1154. [PubMed: 18363838] 
11. Paknikar KM, Jeremic A. Discovery of the cell secretion machinery. J Biomed Nanotechnol. 2007; 3:218-222.

12. Paknikar KM. Landmark discoveries inintracellular transport and secretion. J Cell Mol Med. 2007; 11:393-397. [PubMed: 17635635]

13. Wheatley DN. Pores for thought: further landmarks in the elucidation of the mechanism of secretion. Cell Biol Int. 2007; 31:1297-1300. [PubMed: 17698378]

14. Labhasetwar V. A milestone in science: discovery of the porosome - the universal secretory machinery in cells. J Biomed Nanotechnol. 2007; 3:1.

15. Allison DP, Doktyez MJ. Cell secretion studies by force microscopy. J Cell Mol Med. 2006; 10:847-856. [PubMed: 17125589]

16. Anderson LL. Cell secretion - finally sees the light. J Cell Mol Med. 2006; 10:270-272.

17. Jeftinija S. The story of cell secretion: events leading to the discovery of the 'porosome'-the universal secretory machinery in cells. J Cell Mol Med. 2006; 10:273-279. [PubMed: 16796798]

18. Leabu M. Discovery of the molecular machinery and mechanisms of membrane fusion in cells. $J$ Cell Mol Med. 2006; 10:423-427. [PubMed: 16796809]

19. Anderson LL. Discovery of the 'porosome'; the universal secretory machinery in cells. J Cell Mol Med. 2006; 10:126-131. [PubMed: 16563225]

20. Zhvania MG, Lazrishvili IL. Discovery of a new cellular structure - Porosome. Tsitologiia. 2005; 47:23-27. [PubMed: 16602239]

21. Milosacic N, Prodanovic R. Porosome a new cell structure. Chem Rev. 2004; 45:102-103.

22. Craciun C. Elucidation of cell secretion: pancreas led the way. Pancreatology. 2004; 4:487-489. [PubMed: 15316223]

23. Singer MV. Legacy of a distinguished scientist: George E. Palade, Pancreatology. 2004; 3:518519. [PubMed: 14730177]

24. Zhvania M. The discovery of the molecular mechanism of cellular secretion. J Biol Phys Chem. 2004; 4:43-46.

25. Wheatley DN. A new frontier in cell biology: nano cell biology. Cell Biol Int. 2004; 28:1-2. [PubMed: 14759763]

26. Anderson LL. Discovery of a new cellular structure the porosome: elucidation of the molecular mechanism of secretion. Cell Biol Int. 2004; 28:3-5. [PubMed: 14994739]

27. Hörber JKH, Miles MJ. Scanning probe evolution in biology. Science. 2003; 302:1002-1005. [PubMed: 14605360]

28. Fernandez JM. Cellular and molecular mechanics by atomic force microscopy: Capturing the exocytotic fusion pore in vivo? Proc Natl Acad Sci USA. 1997; 94:9-10. [PubMed: 8990151]

29. Holden C. Early peek at a cellular porthole. Science. 1997; 275:485.

30. Hoffman M. Cell secretion: it's in the pits. Am Sci. 1997; 85:123-124.

31. George KH. New microscope see on the nanoscale. J NIH Res. 1997; 9:32-34.

32. Dufresne DJ. Yale team believes its AFM has imaged elusive fusion pores. Biophotonics Int. 1997 Mar-Apr;:30-31.

33. Joiner KA, Ross DS. Secretory traffic in the eukaryotic parasite Toxoplasma gondii: less is more. J Cell Biol. 2002; 157:557-563. [PubMed: 12011107]

34. Hausmann K, Allen RD. Membranes and microtubules of the excretory apparatus of Paramecium caudatum. Cytobiologie. 1977; 15:303-320.

35. Kubori T, Matsushima Y, Nakamura D, Uralil J, Lara-Tejero M, Sukhan A, Galán JE, Aizawa S-I. Supramolecular structure of the Salmonella typhimurium type III protein secretion system. Science. 1998; 280:602-605. [PubMed: 9554854]

36. White JG. Platelet secretory process. Blood. 1999; 93:2422-2425. [PubMed: 10215351]

37. White JG, Clawson CC. The surface-connected canalicular system of blood platelets -a fenestrated membrane system. Am, J Pathol. 1980; 101:353-359. [PubMed: 7435543]

38. Oyler GA, Higgins GA, Hart RA, Battenberg E, Billingsley M, Bloom FE, Wilson MC. The identification of a novel synaptosomal-associated protein, SNAP-25, differentially expressed by neuronal subpopulations. J Cell Biol. 1989; 109:3039-3052. [PubMed: 2592413] 
39. Bennett MK, Calakos N, Scheller RH. Syntaxin: A synaptic protein implicated in docking of synaptic vesicles at presynaptic active zones. Science. 1992; 257:255-259. [PubMed: 1321498]

40. Trimble WS, Cowan DW, Scheller RH. VAMP-1: A synaptic vesicle-associated integral membrane protein. Proc Natl Acad Sci USA. 1988; 85:4538-4542. [PubMed: 3380805]

41. Malhotra V, Orci L, Glick BS, Block MR, Rothman JE. Role of an N-ethylmaleimide-sensitive transport component in promoting fusion of transport vesicles with cisternae of the Golgi stack. Cell. 1988; 54:221-227. [PubMed: 3390865]

42. Wilson DW, Whiteheart SW, Wiedmann M, Brunner M, Rothman JE. A multisubunit particle implicated in membrane fusion. J Cell Biol. 1992; 117:531-538. [PubMed: 1315316]

43. Sutton RB, Fasshauer D, Jahn R, Brunger AT. Crystal structure of a SNARE complex involved in synaptic exocytosis at $2.4 \AA$ resolution. Nature. 1998; 395:347-353. [PubMed: 9759724]

44. Cho S-J, Kelly M, Rognlien KT, Cho J, Hörber JK, Jena BP. SNAREs in opposing bilayers interact in a circular array to form conducting pores. Biophys, J. 2002; 83:2522-2527. [PubMed: 12414686]

45. Cook JD, Cho WJ, Stemmler TL, Jena BP. Circular dichroism (CD) spectroscopy of the assembly and disassembly of SNAREs: the proteins involved in membrane fusion in cells. Chem Phys Lett. 2008; 462:6-9. [PubMed: 19412345]

46. Jeremic A, Kelly M, Cho J, Cho SJ, Hörber JK, Jena BP. Calcium drives fusion of SNAREapposed bilayers. Cell Biol Int. 2004; 28:19-31. [PubMed: 14759765]

47. Jeremic A, Cho WJ, Jena BP. Membrane fusion: what may transpire at the atomic level. J Biol Phys \& Chem. 2004; 4:139-142.

48. Potoff JJ, Issa Z, Manke CW Jr, Jena BP. Ca2+-Dimethylphosphate complex formation: providing insight into $\mathrm{Ca} 2+$ mediated local dehydration and membrane fusion in cells. Cell Biol Int. 2008; 32:361-366. [PubMed: 18452809]

49. Siksou L, Rostaing P, Lechaire J-P, Boudier T, Ohtsuka T, Fejtova A, Kao H-T, Greengard P, Gundelfinger ED, Triller A, Marty S. Three-dimensional architecture of presynaptic terminal cytomatrix. J Neurosci. 2007; 27:6868-6877. [PubMed: 17596435]

50. Larina O, Bhat P, Pickett JA, Launikonis BS, Shah A, Kruger WA, Edwardson MJ, Thorn P. Dynamic regulation of the large exocytic fusion pore in pancreatic acinar cells. Mol Biol Cell. 2007; 18:3502-3511. [PubMed: 17596517]

51. Savigny P, Evans J, McGarth KM. Cell membrane structures during exocytosis. Endocrinology. 2007; 148:3863-3874. [PubMed: 17494998]

52. Cho WJ, Ren G, Jena BP. EM 3D contourmaps provide protein assembly at the nanoscale within the neuronal porosome complex. J Microscopy. 2008; 232:106-111.

53. Taraska JW, Perrais D, Ohara-Imaizumi M, Nagamatsu S, Almers W. Secretory granules are recaptured largely intact after stimulated exocytosis in cultured endocrine cells. Proc Natl Acad Sci USA. 2003; 100:2070-2075. [PubMed: 12538853]

54. Aravanis AM, Pyle JL, Tsien RW. Single synaptic vesicles fusing transiently and successively without loss of identity. Nature. 2003; 423:643-647. [PubMed: 12789339]

55. Thorn P, Fogarty KE, Parker I. Zymogen granule exocytosis is characterized by long fusion pore openings and preservation of vesicle lipid identity. Proc Natl Acad Sci USA. 2004; 101:67746779. [PubMed: 15090649]

56. Lee JS, Mayes MS, Stromer MH, Scanes CG, Jeftinija S, Anderson LL. Number of secretory vesicles in growth hormone cells of the pituitary remains unchanged after secretion. Exp Biol Med. 2004; 229:291-302.

57. Cohen FS, Niles WD. Reconstituting channels into planar membranes: a conceptual framework and methods for fusing vesicles to planar bilayer phospholipid membranes. Methods in Enzymology. 1993; 220:50-68. [PubMed: 7688845]

58. Woodbury DJ. Nystatin/ergosterol method for reconstituting ion channels into planar lipid bilayers. Methods in Enzymology. 1999; 294:319-339. [PubMed: 9916236]

59. Kelly ML, Woodbury DJ. Ion channels from synaptic vesicle membrane fragments reconstituted into lipid bilayers. Biophys J. 1996; 70:2593-2599. [PubMed: 8744298]

60. Woodbury DJ, Miller C. Nystatin-induced liposome fusion. A versatile approach to ion channel reconstitution into planar bilayers. Biophys J. 1990; 58:833-839. [PubMed: 1701101] 
61. Cho WJ, Jeremic A, Jena BP. Size of supramolecular SNARE complex membrane-directed selfassembly. J Am Chem Soc. 2005; 127:10156-10157. [PubMed: 16028912]

62. Jeremic A, Quinn AS, Cho WJ, Taatjes DJ, Jena BP. Energy-dependent disassembly of selfassembled SNARE complex: observation at nanometer resolution using atomic force microscopy. J Am Chem Soc. 2006; 128:26-27. [PubMed: 16390104]

63. Cho WJ, Jena BP. N-ethymaleimide sensitive factor is a right-handed molecular motor. J Biomed Nanotech. 2007; 3:209-211.

64. Gaisano HY, Sheu L, Wong PP, Klip A, Trimble WS. SNAP-23 is located in the basolateral plasma membrane of rat pancreatic acinar cells. FEBS Lett. 1997; 414:298-302. [PubMed: 9315706]

65. Bennett V. Spectrin-based membrane skeleton: a multipotential adaptor between plasma membrane and cytoplasm. Physiol Rev. 1990; 70:1029-1065. [PubMed: 2271059]

66. Faigle W, Colucci-Guyon E, Louvard D, Amigorena S, Galli T. Vimentin filaments in fibroblasts are a reservoir for SNAP-23, a component of the membrane fusion machinery. Mol Biol Cell. 2000; 11:3485-3494. [PubMed: 11029050]

67. Goodson HV, Valetti C, Kreis TE. Motors and membrane traffic. Curr Opin Cell Biol. 1997; 9:1828. [PubMed: 9013678]

68. Nakano M, Nogami S, Sato S, Terano A, Shirataki H. Interaction of syntaxin with a-fodrin, a major component of the submembranous cytoskeleton. Biochem Biophys Res Commun. 2001; 288:468-475. [PubMed: 11606066]

69. Ohyama A, Komiya Y, Igarashi M. Globular tail of myosin-V is bound to vamp/synaptobrevin. Biochem Biophys Res Commun. 2001; 280:988-991. [PubMed: 11162623]

70. Cho WJ, Jeremic A, Jena BP. Direct interaction between SNAP-23 and L-type calcium channel. J Cell Mol Med. 2005; 9:380-386. [PubMed: 15963257]

71. Jeong EH, Webster P, Khuong CQ, Abdus Sattar AK, Satchi M, Jena BP. The native membrane fusion machinery in cells. Cell Biol Int. 1999; 22:657-670. [PubMed: 10452836]

72. Bako I, Hutter J, Palinkas G. Car-Parrinello molecular dynamics simulation of the hydrated calcium ion. J Chem Phys. 2002; 117:9838-9843. 

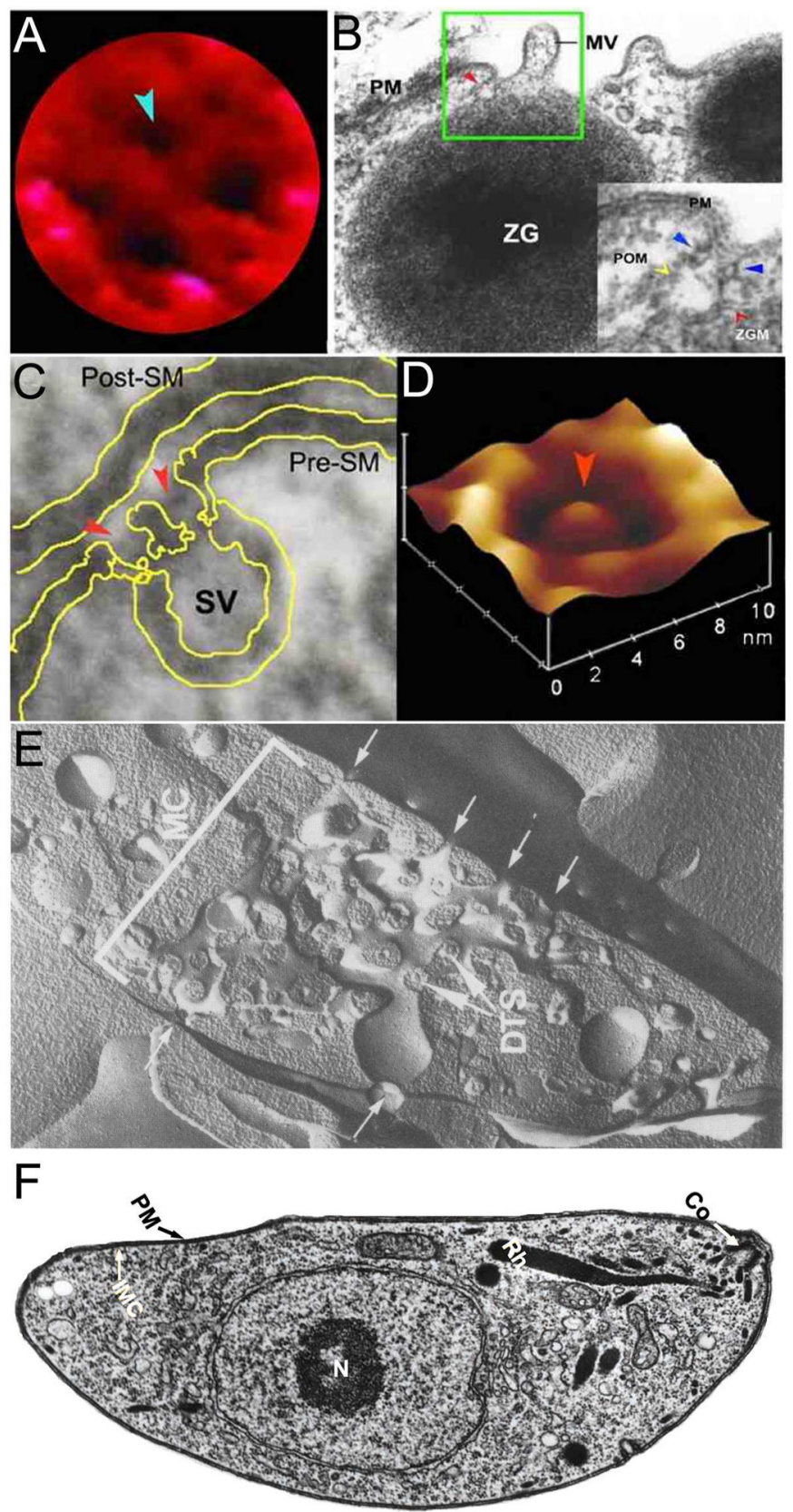

Figure 1.

Porosomes, previously referred to as 'depression' at the plasma membrane in pancreatic acinar cell and at the nerve terminal. (A) A high resolution AFM micrograph shows a single pit with four 100-180 nm porosomes within (blue arrowhead) at the apical plasma membrane in a live pancreatic acinar cell. (B) An electron micrograph depicting a porosome (red arrowhead) close to a microvilli (MV) at the apical plasma membrane (PM) of a pancreatic acinar cell. Note the association of the porosome membrane (yellow arrowhead), and the zymogen granule membrane (ZGM) (red arrow head) of a docked ZG (inset). Cross section of a circular complex at the mouth of the porosome is seen (blue arrow head). (C) Electron micrograph of a porosome (red arrowhead) at the nerve terminal, in association 
with a synaptic vesicle (SV) at the presynaptic membrane (Pre-SM). Notice the central pluglike structure at the neuronal porosome opening. (D) AFM micrograph of a neuronal porosome in physiological buffer, also showing the central plug (red arrowhead) at its opening. The central plug in the neuronal porosome complex may regulate its rapid closeopen conformation during neurotransmitter release. The neuronal porosome is an order of magnitude smaller (10-15 nm) compared to porosome in the exocrine pancreas (22). (E) Electron micrograph of a freeze-fractured discoid platelet. The organization and intercommunication of open calaliculi system (OCS) demonstrating docked and fused secretory vesicles. Several apertures (T) of the OCS open into a communicating web of fenestrated channels that stretches across the cytoplasm to openings ( $t$ ) at the cell membrane. Elements of the dense tubular system (DTS) are interwoven with channels of the OCS in membrane complexes (MC) within the platelet (x 27,000). Reprinted from Am J Pathol 1980 101: 353-364 with permission from the American Society for Investigative Pathology (37). (F) T. gondii parasite possesses a stable polarized secretory apparatus and a micropore (right). The secretory apparatus has three morphologically distinct organelles, namely micronemes, rhoptries ( $\mathrm{Rh})$, and dense granules. The conoid $(\mathrm{Co})$ is present at the apical end of the parasite. Note the micropore is continuous with the plasma membrane (PM) of the parasite. The parasite also has an inner membrane complex (IMC). Reprinted with permission: Joiner and Roos, 2004. Originally published in The Journal of Cell Biology. doi: 10.1083/jcb.200112144 (33). 

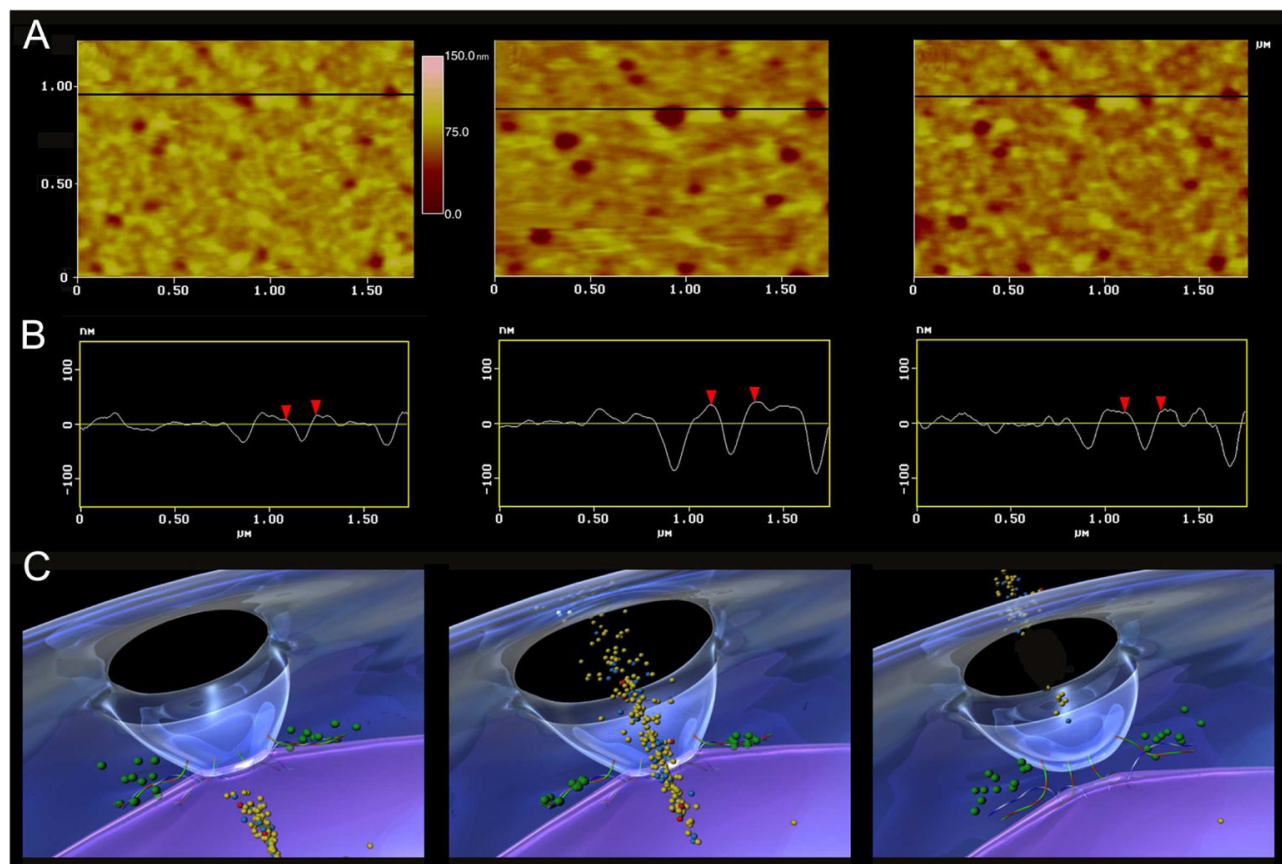

D

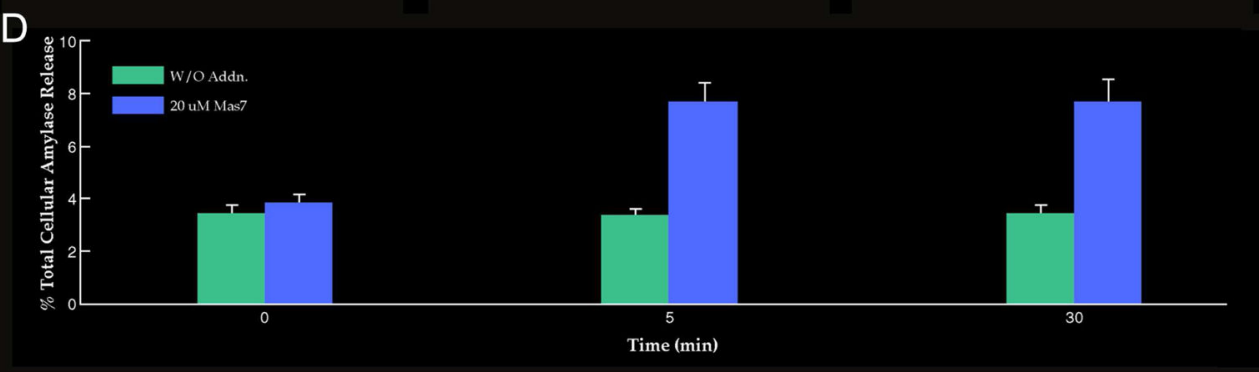

Figure 2.

Porosome dynamics in pancreatic acinar cells following stimulation of cell secretion. (A) Several porosomes within a pit are shown at zero time, $5 \mathrm{~min}$ and $30 \mathrm{~min}$ following stimulation of secretion. (B) Section analysis across three porosomes in the top panel is represented graphically in the second panel and defines the diameter and relative depth of each of the three porosomes. The porosome at the center is represented by red arrow heads. (C) The third panel is a 3D rendition of the porosome complex at different times following stimulation of secretion. Note the porosome as a blue cup-shaped structure with black opening to the outside, and part of a secretory vesicle (violet) docked at its base via t-/vSNAREs. (D) The bottom panel represents \% total cellular amylase release in the presence and absence of the secretagogue Mas7 (blue bars). Note an increase in porosome diameter and relative depth, correlating with an increase in total cellular amylase release at 5 min following stimulation of secretion. At 30 min following a secretory stimulus, there is a decrease in diameter and relative depth of porosomes and no further increase in amylase release beyond the 5-min time point. No significant changes in amylase secretion (green bars) or porosome diameter were observed in control cells in either the presence or absence of the non-stimulatory mastoparan analogue (Mas17). High-resolution images of porosomes were obtained before and after stimulation with Mas7, for up to $30 \mathrm{~min}$ (1). 
A



C
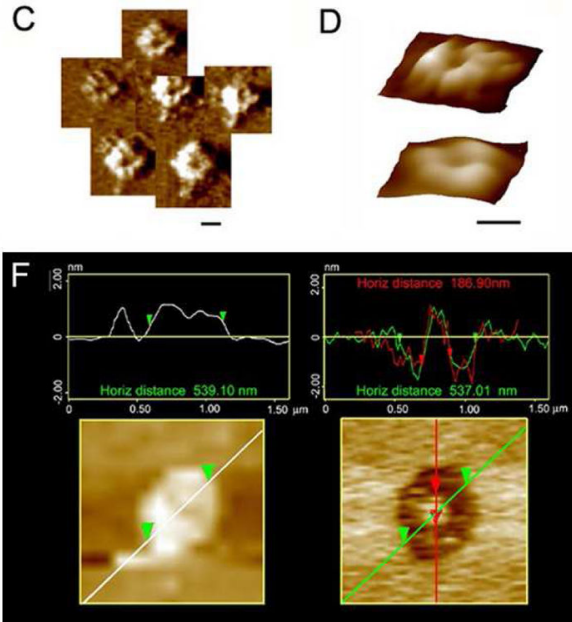

$\mathrm{H}$

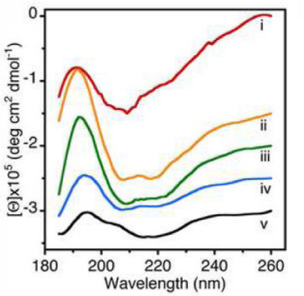

I

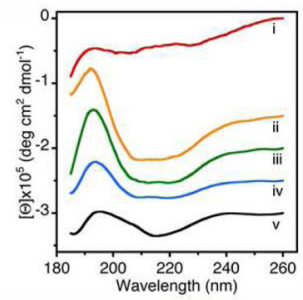

B

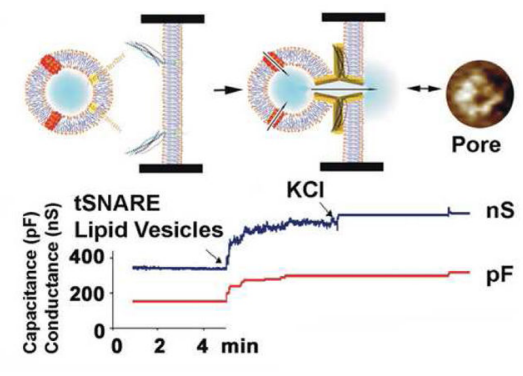

E

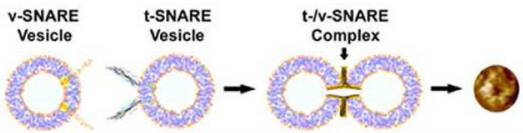

G
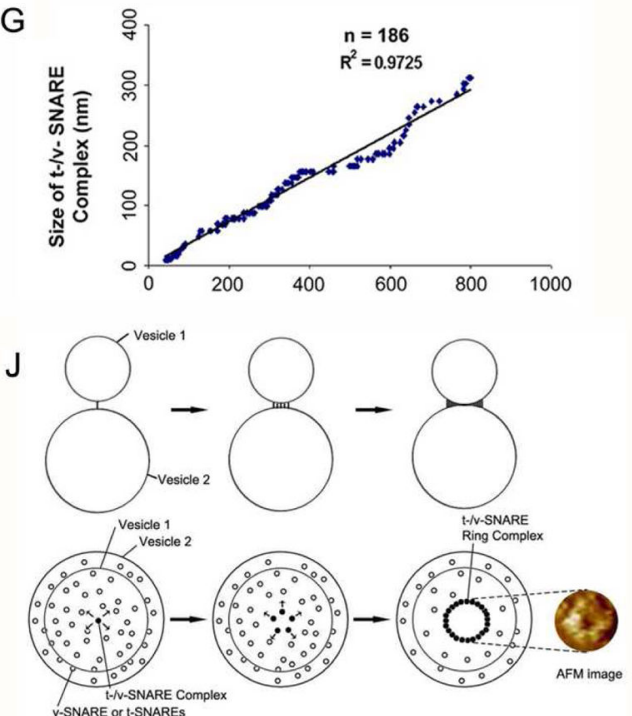

Figure 3.

Membrane-directed assembly and the disassembly of SNAREs. Opposing bilayers containing $\mathrm{t}$ and $\mathrm{v}$-SNAREs respectively, interact in a circular array to form conducting channels in the presence of calcium. (A) Schematic diagram of the bilayer-electrophysiology setup (EPC9). (B) Lipid vesicle containing nystatin channels ( red) and membrane bilayer with SNAREs demonstrate significant changes in capacitance and conductance. When tSNARE vesicles were added to a v-SNARE membrane support, the SNAREs in opposing bilayers arranged in a ring pattern, forming pores as shown in the AFM micrographs (C,D). $\mathrm{t}-\mathrm{v}-\mathrm{SNARE}$ ring complex at low $(C)$ and high resolution $(D)$ is shown. Bar $=100 \mathrm{~nm}$. A stepwise increases in capacitance and conductance $(-60 \mathrm{mV}$ holding potential) is demonstrated following docking and fusion of SNARE-reconstituted vesicles at the SNARE-reconstituted bilayer of the EPC9 electrophysiological set up (B). Docking and fusion of the vesicle at the bilayer membrane opens vesicle-associated nystatin channels and SNARE-induced pore formation, allowing conductance of ions from the cis to the trans side of the bilayer membrane (B). Further addition of $\mathrm{KCl}$ to induce gradient-driven fusion resulted in little or no further increase in conductance and capacitance, demonstrating that docked vesicles have already fused, and that the membrane is intact (B). $(E-G)$ The size of the $t$-/v-SNARE complex is directly proportional to the size of the SNARE-reconstituted 
vesicles. $(E)$ Schematic diagram depicting the interaction of t-SNARE-reconstituted and vSNARE-reconstituted liposomes. $(F)$ AFM images of docked v-SNARE vesicle at t-SNARE reconstituted membrane, before and after its dislodge using the AFM cantilever tip, exposing the t-/v-SNARE-ring complex at the center. $(G)$ Note the high correlation coefficient between vesicle diameter and size of the SNARE complex. (H,I) CD data reflecting structural changes to SNAREs, both in suspension and in association with membrane. Structural changes, following the assembly and disassembly of the t-/v-SNARE complex is further shown. (H) CD spectra of purified full-length SNARE proteins in suspension and (I) in membrane-associated; their assembly and (NSF-ATP)-induced disassembly is demonstrated. (i) v-SNARE; (ii) t-SNAREs; (iii) t-/v-SNARE complex; (iv) $\mathrm{t}-/ \mathrm{v}-\mathrm{SNARE}+\mathrm{NSF}$ and (v) t-/v-SNARE + NSF + $2.5 \mathrm{mM}$ ATP, is shown. CD spectra were recorded at $25^{\circ} \mathrm{C}$ in $5 \mathrm{mM}$ sodium phosphate buffer ( $\mathrm{pH} 7.5$ ), at a protein concentration of $10 \mu \mathrm{M}$. In each experiment, 30 scans were averaged per sample for enhanced signal to noise, and data were acquired on duplicate independent samples to ensure reproducibility (45). (J) Schematic diagram depicting the possible molecular mechanism of SNARE ring complex formation, when t-SNARE-vesicles and V-SNARE-vesicles meet. The process may occur due to a progressive recruitment of $\mathrm{t}-\mathrm{v}$-SNARE pairs as the opposing vesicles are pulled toward each other, until a complete ring is established, preventing any further recruitment of $\mathrm{t}-/ \mathrm{v}-\mathrm{SNARE}$ pairs to the complex. The top panel is a side view of two vesicles (one tSNARE-reconstituted, and the other $v$-SNARE reconstituted) interacting to form a single $\mathrm{t}$-/v-SNARE complex, leading progressively (from left to right) to the formation of the ring complex. The lower panel is a top view of the two interacting vesicles. 

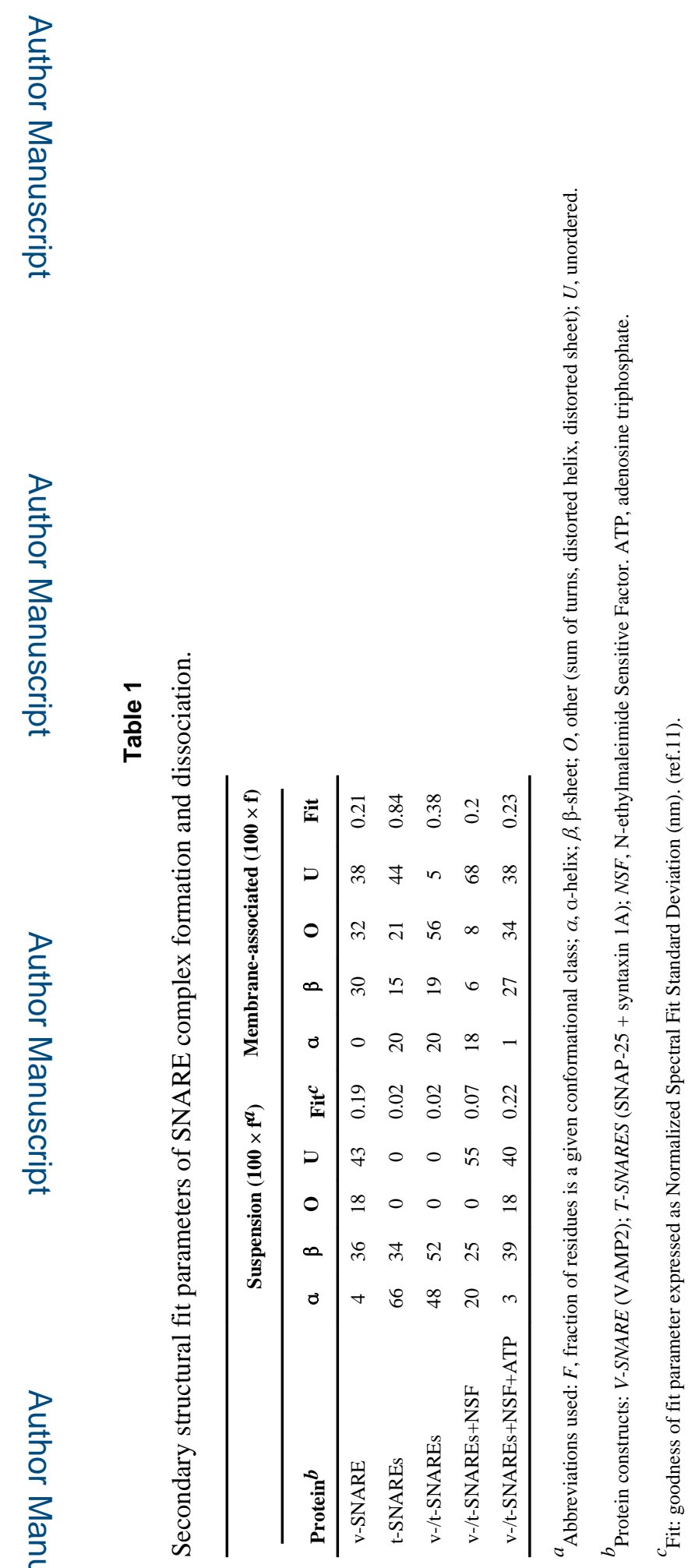

Biochemistry. Author manuscript; available in PMC 2015 September 23. 